\title{
Grußwort des Bergmännischen Verbandes Österreichs
}

\author{
Gerhard Mayer ${ }^{1}$ und Josef Pappenreiter ${ }^{2}$ \\ 'Department Mineral Resources Engineering, Montanuniversität Leoben, Leoben, Österreich \\ ${ }^{2}$ VA Erzberg GmbH, Eisenerz, Österreich
}

Online publiziert 26. Oktober 2020

Mit der Herausgabe von Heft 10/2020 der BHM setzt der Lehrstuhl für Aufbereitung und Veredlung der Montanuniversität Leoben ein Zeichen zu seinem 90-jährigen Bestehen.

Im Bergmännischen Verband Österreichs hat die Fachgruppe der Aufbereiter einen ganz besonderen Stellenwert. Der Lehrstuhl und allen voran dessen Leiter, Herr Univ.Prof. Dipl.-Ing. Dr. mont. Helmut Flachberger, stehen für technisch-wissenschaftlichen Fortschritt und akademische Lehre auf höchstem Niveau. Kaum eine andere Fachgruppe unseres Verbandes zeichnet sich durch einen derartigen $\mathrm{Zu}$ sammenhalt und Elan im Bemühen aus, den eigenen Fachbereich voranzutreiben, der Öffentlichkeit bekannt und für unseren akademischen Nachwuchs interessant zu machen.

Höhepunkt in diesem Bemühen ist aus der Sicht des Bergmännischen Verbandes Österreichs das jährlich stattfindende Aufbereitungstechnische Seminar an der Montanuniversität Leoben, das von unserem Fachausschuss für Aufbereitung gemeinsam mit dem Lehrstuhl für Aufbereitung und Veredlung fachlich geprägt wird. Diese hochkarätige und international besetzte Leistungsschau des Lehrstuhls und der mit ihm verbundenen Industrie wird seit dem Jahr 2003 regelmäßig ausgerichtet und dient neben dem jeweiligen fachlichen Schwerpunkt auch dem Brückenschlag zwischen der Universität, der Industrie und unserer Jugend.

Eine derart erfolgreiche und über viele Jahrzehnte reichende positive Entwicklung braucht neben der wissenschaftlichen Leitung auch das Geschick, viele begabte und fachlich engagierte Mitarbeiter um sich zu scharen. All das ist nachhaltig gelungen.
Der Bergmännische Verband Österreichs spricht dem Lehrstuhl für Aufbereitung und Veredlung dafür Dank und Anerkennung aus. Wir wünschen weiterhin viel Erfolg.

Qualität hat Bestand!

\section{Gerhard Mayer \\ Geschäftsführer}

Josef Pappenreiter

Präsident

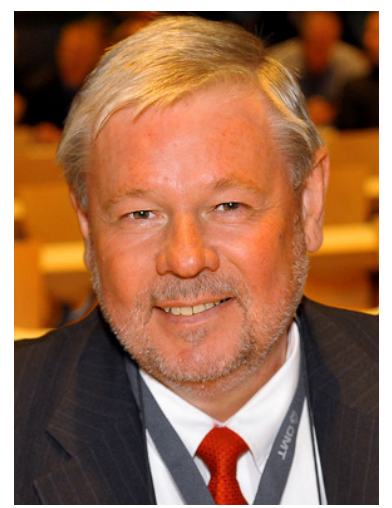

Gerhard Mayer

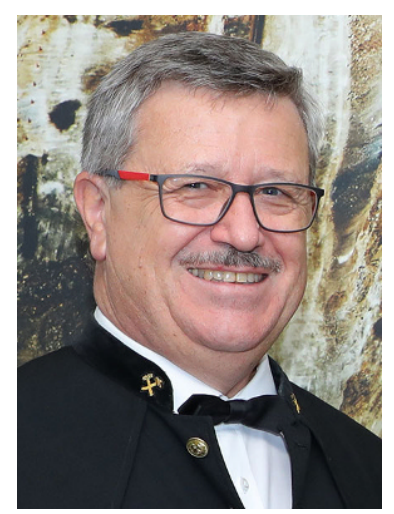

Josef Pappenreiter 